\title{
WHOLE-GENOME SEQUENCING IN RELATION TO RESISTANCE OF MYCOBACTERIUM TUBERCULOSIS
}

\author{
DOHAL M ${ }^{1}$, PORVAZNIK I ${ }^{2,3}$, KUSNIR P ${ }^{1}$, MOKRY J. ${ }^{1}$
}

${ }^{1}$ Department of Pharmacology and Biomedical Center Martin, Jessenius Faculty of Medicine in Martin, Comenius University in Bratislava, Martin, Slovak Republic, ${ }^{2}$ Department of Clinical Microbiology, National Institute for Tuberculosis, Lung Diseases and Thoracic Surgery, Vyšné Hágy, Slovak Republic, ${ }^{3}$ Faculty of Health, Catholic University, Ružomberok, Slovak Republic

\section{Abstract}

Tuberculosis, a disease caused by Mycobacterium tuberculosis, represents one of the deadliest infections worldwide. The incidence of resistant forms is increasing year by year; therefore, it is necessary to involve new methods for rapid diagnostics and treatment. One of the possible solutions is the use of whole-genome sequencing (WGS).

The WGS provides an identification of complete genome of the microorganism, including all genes responsible for resistance, in comparison with other genotypic methods (eg. Xpert MTB / RIF or Hain line-probes) that are capable to detect only basic genes. WGS data are available in 1-9 days and several online software tools (TBProfiler, CASTB, Mykrobe PredictorTB) are used for their interpretation and analysis, compared to 3-8 weeks in the case of classic phenotypic evaluation.

Furthermore, WGS predicts resistance to the first-line antituberculotics with a sensitivity of $85-100 \%$ and a specificity of $85-100 \%$.

This review elucidates the importance and summarizes the current knowledge about the possible use of WGS in diagnosis and treatment of resistant forms of tuberculosis elucidates.

WGS of M. tuberculosis brings new possibilities for rapid and accurate diagnostics of resistant forms of tuberculosis. Introducing WGS into routine practice can help to reduce the spread of resistant forms of tuberculosis as well as to increase the success rate of the treatment, especially through an appropriate combination of antituberculotics ATs. Introduction of WGS into routine diagnostics can, in spite of the financial difficulty, significantly improve patient care.

Keywords: whole-genome sequencing, mycobacterium tuberculosis, drug resistance

\section{INTRODUCTION}

Mycobacterium (M.) tuberculosis is an acid-resistant intracellular bacterium which is known as causative agent of tuberculosis (TB). According to World Health Organisation (WHO) 1.7 milion people died from the disease in 2016 (including 400,000 among people with HIV) therefore nowadays tuberculosis is among the most common causes of death worldwide. However, even more disturbing is the emergence of drug resistance mostly to the first-line ATs, which significantly complicates the treatment. It is assumed that more than 50 million people are infected with resistant strains of $M$. tuberculosis worldwide. The most frequently is diagnosed is multi drug-resistant tuberculosis (MDR-TB - 4\% of all new cases), followed by extensively drug-resistant tuberculosis (XDR-TB) and during last years new cases of totally drug-resistant tuberculosis (TDR-TB) were detected (1). Despite the fact that incidence of tuberculosis in Slovakia is low, actual social changes in the community, cessation with vaccinations, as well as an increasing number of migrants may worsen the situation (Fig. 1).

\section{Corresponding author:}

Mgr. Matúš Dohál, Department of Pharmacology, Jessenius Faculty of Medicine, Comenius University, Malá Hora 4C, 03601 Martin;

E-mail: matus.dohal1@gmail.com; Phone: +421 432633631 


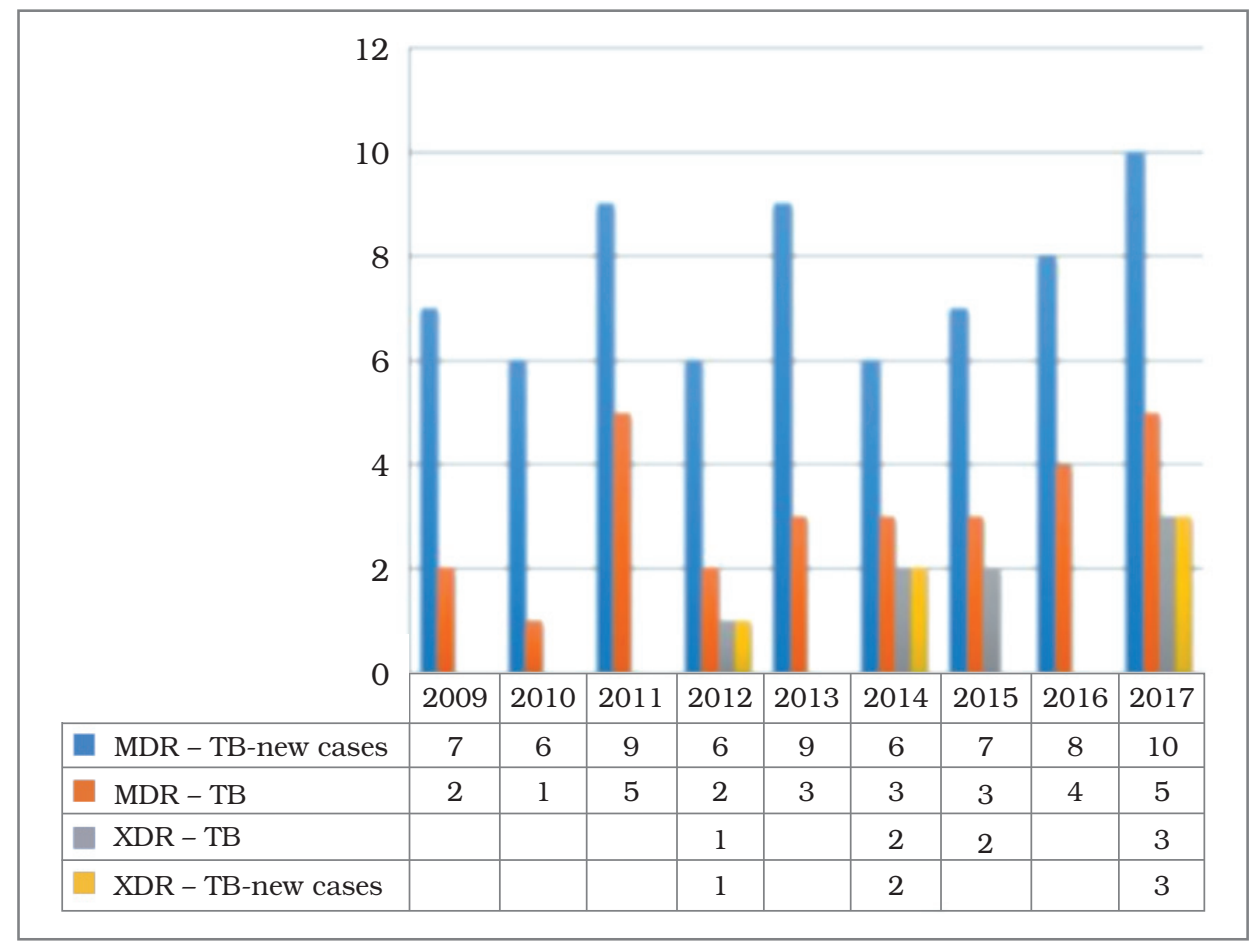

Fig. 1: The incidence of MDR and XDR - TB in Slovakia in the years of 1999-2017

Resistant TB represents a huge problem with a hopeless solution mainly in developing countries. For stopping this global threat an accurate and fast microbiological diagnostics along with the search for infected patients and their appropriate treatment is necessary. The diagnostics is based on an isolation of M. tuberculosis from biological material, identification of the strain, and determination of sensitivity to antituberculotics or direct determination of resistance to isoniazid, rifampicin, etambutol, aminoglycosides, cyclic peptides, and fluoroquinolones by molecular-genetic methods $(2 ; 3)$.

According to WHO recommendations, the treatment of TB should include a combination of five effective drugs. However, it is not possible to achieve the effectiveness of the drug combination in many cases, especially because of the resistance or patient's intolerance to the drugs $(4 ; 5)$. The overall success rate of TB treatment is $83 \%$, which significantly reduces the incidence of resistant forms of bacteria. On the other hand, the treatment efficacy in MDR-TB patients is only 54\%, whereas in patients treated for XDR-TB, the success rate is only $30 \%$ (6). Early diagnostics and the appropriate treatment may reduce spreading and development of resistant forms of TB; however, a low availability of fast diagnostic methods still generates a huge problem $(7 ; 8)$. The WGS method is an alternative that could be used in diagnostic practice in near future as it overcomes the limitations of the currently used phenotypic and genotypic methods (9).

The goal of this review is to elucidate the importance and to summarize the current knowledge about the possible use of WGS in diagnostics and treatment of resistant forms of tuberculosis.

\section{TUBERCULOSIS GENOME}

The genome of $M$. tuberculosis (H37Rv strain) was completely sequenced for the first time in 1998. It contains approximately 4.4 million base pairs encoding 4,000 genes (most of which are involved in lipolysis and lipogenesis) and a high content of $\mathrm{G}+\mathrm{C}$ pairs (65.6\%) (10) (Fig. 2). 


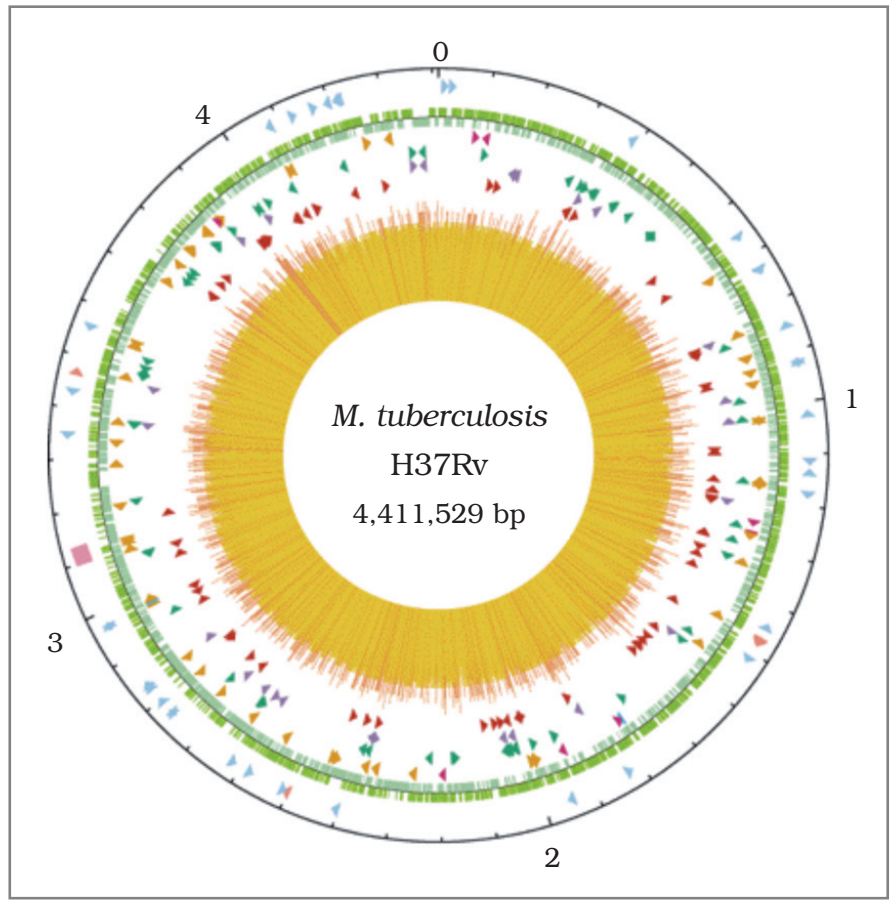

Fig. 2: Structure of $M$. tuberculosis genome (Cole et al., 1998)

It has been assumed that the genetic variability between the individual strains is minimal and has no clinical significance. An important role in defeating this dogma played the WGS method, as it has been used for identification of a large number of strains. Furthermore, it has been confirmed that genomic variations are important in their phenotypic properties (e.g., increased virulence in the family of Beijing tribes) $(11 ; 12 ; 13)$.

\section{WHOLE-GENOME SEQUENCING (WGS)}

The WGS method provides an identification of the complete DNA sequence (genome) of the organism at a particular time. It includes sequencing the chromosomal DNA, mitochondrial DNA, and, in the case of plants, also of DNA contained in chloroplasts. Technology development over the past 15 years has dramatically reduced the complexity, cost, and sequencing time. The mostly used sequencing platforms include IonTorrent, Illumina, and SMRT (14). The first step before the sequencing is a preparation of the sample. The DNA sample is split into fragments of the same length. The ends of the individual fragments are enzymatically edited to bind the respective adapters. Fragments with a length of $200-500 \mathrm{bp}$ are selected for sequencing by the electrophoretic separation (these steps are so-called preparation of library). The last step before the sequencing is an amplification of DNA fragments which is usually done by the bridge polymer chain reaction (PCR). During the sequencing each of the four free deoxynucleotides (dNTPs) is labeled with a special fluorescent dye (Illumina - labeled dNTP). After incorporation of the nucleotides the fluoroscent signal is measured. The process is repeated until the entire library is captured (15; 16) (Fig. 3).

WGS has a great potential to become an inevitable tool in controlling the antibiotic resistance, the major threat in modern healthcare. WGS has already reached a number of applications in this area. These include the development of new antibiotics, diagnostic tests, or the control of antibiotics by monitoring and clarifying factors that influence the onset and persistence of resistance (17). The portable Minion sequencer (Oxford Nanopore Technologies, United Kingdom) has been extensively used in the diagnosis of Ebola virus cases in West 


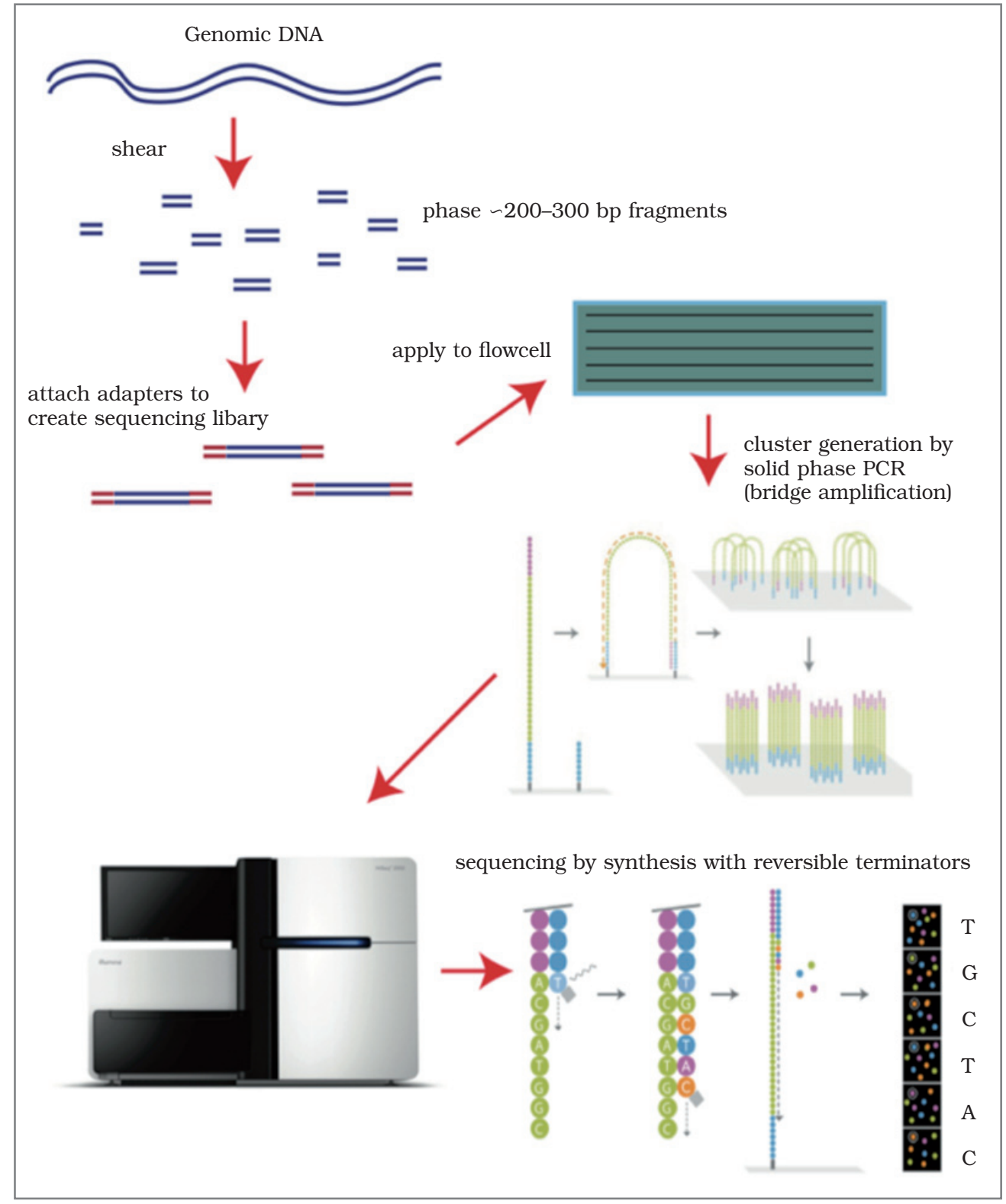

Fig. 3: WGS on Illumina Miseq (source: https://bitesizebio.com/13546/sequencing-by-synthesisexplaining-the-illumina-sequencing-technology/)

Africa and the advantage of this machine lies in its ability to identify pathogens and antimicrobial resistance directly from urine $(18 ; 19)$. Based on the WHO guidelines a network of testing centers and special laboratories has been set up for using WGS to determine HIV virus resistance against anti-retroviral drugs (20).

\section{WGS AND M. TUBERCULOSIS RESISTANCE}

In addition to clinical use in the field of cancer and hereditary diseases, WGS may lead to a breakthrough in the diagnosis of infections caused by $M$. tuberculosis. It enables the iden- 
tification and detection of the susceptibility of the test strain in a clinically relevant timeframe, contrary to conventionally used methods based on phenotypic examination (this may take up to 8 weeks) $(21 ; 22)$ (Fig. 4$)$.

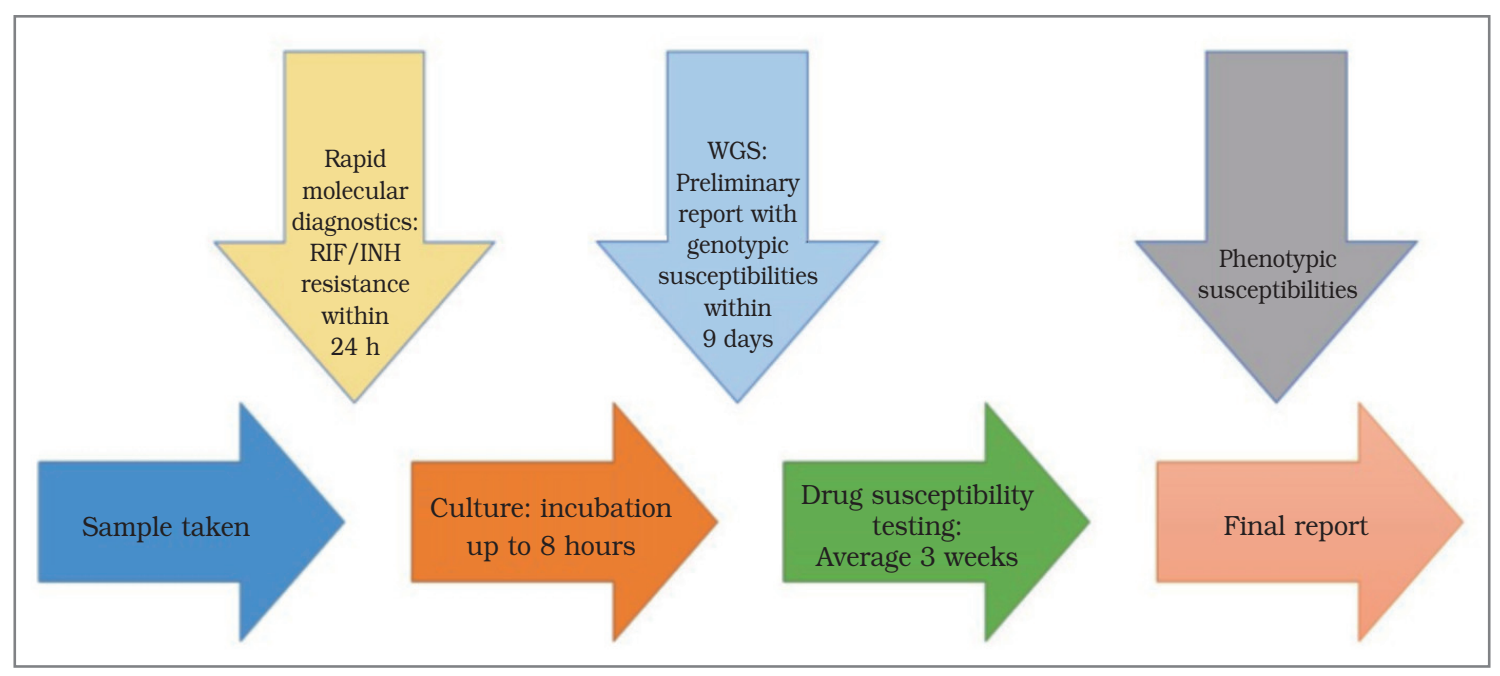

Fig. 4: Comparison of evaluation by genotypic (Xpert, WGS) and phenotypic methods. Results of genotypic methods are available in 1-9 days. In the case of classical phenotypic evaluation, it is 3-8 weeks (according to Satta et al., 2017).

M. tuberculosis resistance is induced due to a large extent single-nucleotide polymorphism (SNP), insertions, deletions, and to a significantly lesser extent to horizontal gene transfer via plasmids or transposons (23). SNPs represent all single nucleotide variants in the $M$. tuberculosis genome when compared to the reference genome, and mutations mean a subset of the SNPs that are relevant or functional concerning the resistance (24). It is assumed that changes in the regulation of efflux pumps also play a role in the development of resistance (25). Currently, there is a number of well described gene mutations and the mechanisms of resistance caused by this mutations (Tab. 1).

The standardized methodology for interpretation of the relationship between mutations and the phenotypic signs of resistance to ATs has been developed recently. Mutations are divided into several groups with a high, intermediate, minimal, or indefinite relationship to resistance or are not associated with resistance at all (26). However, the mechanism of resistance is not known in 10-40\% of resistant strains. It is supposed that SNP are responsible for resistance in most cases.

WGS is used for accurate identification of characterized and non-characterized mutations and significantly supports the continuous development of a reference library of mutations responsible for resistance (e.g. online software "TB profiler") $(27 ; 28)$. Recently, WGS has confirmed that a frequency of new mutations is higher in the TB positive patients with failing treatment compared to the successfully treated patients $(29 ; 30)$. In addition, WGS has also discovered that some mutations previously considered as markers of resistance to etambutol are in fact only markers for a particular phylogenetic $M$. tuberculosis family. For this reason false positive results have often occurred in countries with an occurrence of this phylogenetic family and this knowledge should be considered in developing new genotypic methods determining the sensitivity of bacteria (17).

Progress has been made in interpreting and standardizing the data. There are several online software tools providing a quick interpretation of WGS data. Online databases (ReSeqTB, TB Portal, GenTB) serve as an option to share, interpret, and link genetic results 
Tab. 1: Overview of genes encoding resistance and mechanisms of M. tuberculosis resistance (Bang, 2010)

\begin{tabular}{|c|c|c|c|}
\hline Drug & Gene & Function of the gene & Mechanism of resistance \\
\hline Rifampin & $r p o B$ & $\begin{array}{l}\text { Beta subunit of RNA } \\
\text { polymerase }\end{array}$ & Inhibition of RNA synthesis \\
\hline \multirow[t]{5}{*}{ Isoniazid } & katG & Catalase-peroxidase & $\begin{array}{l}\text { Reduces of ability to activate the prodrug iso- } \\
\text { niazid }\end{array}$ \\
\hline & $\operatorname{inh} A$ & Enoyl ACP reductase & $\begin{array}{l}\text { Reduces the binding of } \mathrm{NADH} \text { to inhA and } \\
\text { attack by isoniazid }\end{array}$ \\
\hline & $\operatorname{ahpC}$ & $\begin{array}{l}\text { Alkyl hydroperoxide } \\
\text { reductase }\end{array}$ & $\begin{array}{l}\text { Overexpression of antioxidant enzyme AphC, } \\
\text { that removes peroxide necessary for isonia- } \\
\text { zid activation }\end{array}$ \\
\hline & $\operatorname{kas} A$ & $\begin{array}{l}\text { Beta-ketoacyl ACP syn- } \\
\text { thase }\end{array}$ & $\begin{array}{l}\text { Overexpression of KasA, involved in fatty } \\
\text { acid and mycolic acid synthesis }\end{array}$ \\
\hline & $n d h$ & NADH dehydrogenase & $\begin{array}{l}\text { Increases the NADH/NAD ratio, competes- } \\
\text { with the binding of isoniazid-NAD to inhA }\end{array}$ \\
\hline Ethambutol & $e m b B$ & Arabinosyl transferase & Decreaces the binding to ethambutol \\
\hline Pyrazinamide & pncA & Pyrazinamidase & $\begin{array}{l}\text { Lack of conversion of pyrazinamide to pyrazi- } \\
\text { noic acid }\end{array}$ \\
\hline Streptomycin & $r p s L$ & S12 ribosomal protein & $\begin{array}{l}\text { Decreased binding of streptomycin to } \mathrm{S} 12 \text { ribo- } \\
\text { somal protein }\end{array}$ \\
\hline Amikacin & rrs & 16S rRNA & $\begin{array}{l}\text { Decreased binding of aminoglycosides to } 16 \mathrm{~S} \\
\text { rRNA }\end{array}$ \\
\hline Kanamycin & rrs & 16S rRNA & $\begin{array}{l}\text { Decreased binding of aminoglycosides to } 16 \mathrm{~S} \\
\text { rRNA }\end{array}$ \\
\hline Capreomycin & tlyA & $2^{-}$-O-metyltransfererase & \\
\hline Fluoroquinolones & $\begin{array}{l}\text { gyrA, } \\
\text { gyrB }\end{array}$ & DNA gyrase & Inhibition of DNA gyrase \\
\hline Ethionamide & $\operatorname{inh} A$ & Enoyl ACP reductase & Inhibition of mycolic acid synthesis \\
\hline
\end{tabular}

with the phenotype of drug resistance and other epidemiological data. These and other databases also allow control of M. tuberculosis resistance at the global level (31).

\section{COMPARISION OF WGS WITH OTHER MOLECULAR-GENETIC METHODS}

Currently used genotyping methods (eg. Xpert MTB / RIF or Hain line-probes) allow in a relatively short time the identification of individual species of mycobacteria and mutations responsible for their resistance (32). However, the number of identified mutations is very limited. This limitations show major disadvantages compared to WGS $(33 ; 34 ; 35 ; 36 ; 37)$.

Xpert / RIF is a genotypic method using PCR to identify M. tuberculosis and to determine rpoB gene mutations and to confirm a resistance to rifampicin. As soon as positive results 
are obtained the treatment of MDR-TB (caused by a strain resistant to rifampicin and isoniazid) is initiated. However, this procedure is often incorrect. In countries like Iran and India up to $33 \%$ of rifampicin-resistant strains do not exhibit resistance to isoniazid and such treatment may lead to the development and spread of MDR-TB $(38 ; 39)$. For this reason, in addition to the Xpert MTB / RIF, the phenotypic evidence of sensitivity must also be obtained in the US (40). A suitable solution may be the use of WGS method as it allows the identification of a number of genes encoding resistance at the same time, thereby leading to the appropriate treatment.

Coll et al. evaluated isolates from different countries of the world to obtain information about sensitivity and specificity of WGS in resistance prediction compared to phenotypic methods. High values of sensitivity and specificity were confirmed for first-line ATs: rifampicin $96.2 \%$ and $98.1 \%$, isoniazid $92.8 \%$ and $100 \%$, ethambutol $88.7 \%$ and $81.7 \%$, and streptomycin $87.1 \%$ and $89,7 \%$ (33). In a study published by Walker et al. the authors showed the following sensitivity and selectivity values: rifampicin $91.7 \%$ and $99.2 \%$, isoniazid $85.2 \%$ and $98.4 \%$, ethambutol $82.3 \%$ and $95.1 \%$, and streptomycin $86.1 \%$ and $99.1 \%$ (41). These results were later verified by a research team from India confirming $100 \%$ sensitivity and 94\% specificity for rifampicin and isoniazid, 100\% sensitivity and 78\% specificity for ethambutol, and $85 \%$ sensitivity and 100\% specificity for streptomycin (42). Shea et al. decided to use WGS for all clinically confirmed cases of tuberculosis in New York. Species identification was determined with an accuracy of 99\%. The compliance rate of resistance determined by WGS compared to culture sensitivity tests was $96 \%$. In addition, the doctors received results with resistance profiles 9 days earlier for the first-line antituberculosis and up to 30 days earlier for the second-line antituberculosis (ethionamide, kanamycin, ofloxacin) (43).

\section{OTHER USE OF WGS}

The greatest potential of WGS for the clinical practise (but also the biggest challenge) is the ability to sequence the individual strains of $M$. tuberculosis directly from clinical isolates without the cultivation. Doughty et al. diagnosed 7 of 8 positive samples from patients. Although the identification of the $M$. tuberculosis species has provided useful information, a large number of non-tuberculous sequences in combination with insufficient depth of coverage (number of reads covering the particular target nucleotide) and depth of sequencing (total amount of sequencing data) make it impossible to predict resistance (44). Therefore, it is necessary to get the highest possible concentration of $M$. tuberculosis DNA in the sputum for the direct evidence of resistance. For this purpose there are designed special RNA templates (RNA baits, SureSelectXT, Agilent, US) (45). The use of these templates led to confirmation of resistance to rifampicin and isoniazid with 95-97\% sensitivity and 98-100\% specificity in 96 hours directly from sputum $(46 ; 47)$.

Tuberculosis caused by resistant strains does not arise only from selective pressure occurring due to inappropriate therapy or at sub-therapeutic doses of the used ATs. XDRTB often develops after several episodes of ineffective treatment, including the use of second-line AT for treating MDR-TB. However, it is important to note that tuberculosis induced by MDR and XDR strains may also be caused by direct infection by resistant strains from the infected patients (48). WGS allows to study the origin and transmission of resistant forms, particularly in high burden TB countries (49).

Historically it was believed that patients were infected with only one strain of $M$. tuberculosis. This assumption was rebutted by the development of molecular epidemiology technology. WGS has confirmed that TB is a disease caused in many cases by a complex of different strains (50).

In addition to the above-mentioned possibilities of usage, the WGS is a tool for identifying target sites of AT (e.g. bedaquilin drug target) and for patients with recurrent infections to determine whether it is a relapse (patient infected with the same strain) or reinfection (patient infected with a new strain) $(51 ; 52)$. 


\section{CONCLUSIONS}

Whole genome sequencing of $M$. tuberculosis brings new possibilities for rapid and accurate diagnostics of resistant forms of tuberculosis. Introducing WGS into routine practice can help to reduce the spread of resistant forms of tuberculosis as well as to increase the success rate of the treatment, especially through an appropriate combination of ATs. The introduction of WGS into routine diagnostics can, in spite of the financial difficulty, significantly improve patient care.

Acknowledgements: This study was supported by grant APVV-18-0084

\section{REFERENCES}

1. World Health Organisation. Global Tuberculosis Report 2017. Geneva: WHO; 2017.

2. Brossier F, Guindo D, Pham A, et al. Performance of the New Version (v2.0) of the GenoType MTBDRsl Test for Detection of Resistance to Second-Line Drugs in Multidrug-Resistant Mycobacterium tuberculosis Complex Strains. J Clin Microbiol. 2016;54(6):1573-1580.

3. Porvaznik I, Mokry J, Solovic I. Drug resistance to anti-tuberculotics in children - three years status in Slovakia. Acta Medica Martiniana 2013, 13(3): 18-22

4. Dheda K, Gumbo T, Maartens G, et al. The epidemiology, pathogenesis, transmission, diagnosis, and management of multidrug-resistant, extensively drug-resistant, and incurable tuberculosis. Lancet Respir Med. 2017;

5. World Health Organisation. WHO treatment guidelines for drug-resistant tuberculosis 2016 update. Geneva, Switzerland 2016

6. World Health Organisation. Global Tuberculosis Report 2017. Geneva: WHO; 2017.

7. Daley CL, Caminero JA. Management of multidrug resistant tuberculosis. Semin Respir Crit Care Med. 2013;34(1):44-59.

8. Lynch JB. Multidrug-resistant Tuberculosis. Med Clin North Am. 2013;97(4):553-79.

9. Cirillo DM, Miotto P, Tortoli E. Evolution of Phenotypic and Molecular Drug Susceptibility Testing. Adv Exp Med Biol. 2017;1019:221-246.

10. Cole ST, Brosch R, Parkhill J, et al. Deciphering the biology of Mycobacterium tuberculosis from the complete genome sequence. Nature. 1998;393(6685):537-44.

11. Black PA, De vos M, Louw GE, et al. Whole genome sequencing reveals genomic heterogeneity and antibiotic purification in Mycobacterium tuberculosis isolates. BMC Genomics. 2015;16:857.

12. Galagan JE. Genomic insights into tuberculosis. Nat Rev Genet. 2014;15(5):307-20.

13. Niemann S, Supply P. Diversity and evolution of Mycobacterium tuberculosis: moving to wholegenome-based approaches. Cold Spring Harb Perspect Med. 2014;4(12):a021188.

14. Mukhopadhyay R. DNA sequencers: the next generation. Anal Chem. 2009;81(5):1736-40.

15. Anderson MW, Schrijver I. Next generation DNA sequencing and the future of genomic medicine. Genes (Basel). 2010;1(1):38-69.

16. Zvárová J, Mazura I. Metody molekulární biologie a bioinformatiky. 1. vyd. Praha: Karolinum, 2012. Biomedicínská informatika. ISBN 978-80-246-2150-0.

17. Köser CU, Ellington MJ, Peacock SJ. Whole-genome sequencing to control antimicrobial resistance. Trends Genet. 2014;30(9):401-7.

18. Quick J, Loman NJ, Duraffour S, et al. Real-time, portable genome sequencing for Ebola surveillance. Nature. 2016;530(7589):228-232.

19. Schmidt K, Mwaigwisya S, Crossman LC, et al. Identification of bacterial pathogens and antimicrobial resistance directly from clinical urines by nanopore-based metagenomic sequencing. J Antimicrob Chemother. 2017;72(1):104-114.

20. World Health Organisation. HIV drug resistance surveillance guidance: 2015 update, Geneva 2015

21. Pfyffer GE, Wittwer F. Incubation time of mycobacterial cultures: how long is long enough to issue a final negative report to the clinician?. J Clin Microbiol. 2012;50(12):4188-9. 
22. Witney AA, Cosgrove CA, Arnold A, Hinds J, Stoker NG, Butcher PD. Clinical use of whole genome sequencing for Mycobacterium tuberculosis. BMC Med. 2016;14:46.

23. Almeida da silva PE, Palomino JC. Molecular basis and mechanisms of drug resistance in Mycobacterium tuberculosis: classical and new drugs. J Antimicrob Chemother. 2011;66(7):1417-30.

24. Reece RJ. Analysis of Genes and Genomes. Wiley; 2004.

25. De vos M, Müller B, Borrell S, et al. Putative compensatory mutations in the rpoC gene of rifampin-resistant Mycobacterium tuberculosis are associated with ongoing transmission. Antimicrob Agents Chemother. 2013;57(2):827-32.

26. Miotto P, Tessema B, Tagliani E, et al. A standardised method for interpreting the association between mutations and phenotypic drug resistance in . Eur Respir J. 2017;50(6)

27. Farhat MR, Shapiro BJ, Kieser KJ, et al. Genomic analysis identifies targets of convergent positive selection in drug-resistant Mycobacterium tuberculosis. Nat Genet. 2013;45(10):1183-9.

28. Zhang H, Li D, Zhao L, et al. Genome sequencing of 161 Mycobacterium tuberculosis isolates from China identifies genes and intergenic regions associated with drug resistance. Nat Genet. 2013; 45(10):1255-60.

29. Hoagland DT, Liu J, Lee RB, Lee RE. New agents for the treatment of drug-resistant Mycobacterium tuberculosis. Adv Drug Deliv Rev. 2016;102:55-72.

30. Warner DF, Koch A, Mizrahi V. Diversity and disease pathogenesis in Mycobacterium tuberculosis. Trends Microbiol. 2015;23(1):14-21.

31. Satta G, Lipman M, Smith GP, Arnold C, Kon OM, Mchugh TD. Mycobacterium tuberculosis and whole-genome sequencing: how close are we to unleashing its full potential?. Clin Microbiol Infect. 2018;24(6):604-609.

32. Porvaznik I, Mokry J, Solovic I. Classical against molecular-genetic methods for susceptibility testing of antituberculotics. Adv Exp Med Biol. 2015,835:15-22

33. Didelot X, Bowden R, Wilson DJ, Peto TEA, Crook DW. Transforming clinical microbiology with bacterial genome sequencing. Nat Rev Genet. 2012;13(9):601-612.

34. Coll F, Mcnerney R, Preston MD, et al. Rapid determination of anti-tuberculosis drug resistance from whole-genome sequences. Genome Med. 2015;7(1):51.

35. Feuerriegel S, Schleusener V, Beckert P, et al. PhyResSE: a Web Tool Delineating Mycobacterium tuberculosis Antibiotic Resistance and Lineage from Whole-Genome Sequencing Data. J Clin Microbiol. 2015;53(6):1908-14.

36. Parrish N, Carrol K. Importance of improved TB diagnostics in addressing the extensively drugresistant TB crisis. Future Microbiol. 2008;3(4):405-13.

37. Witney AA, Gould KA, Arnold A, et al. Clinical application of whole-genome sequencing to inform treatment for multidrug-resistant tuberculosis cases. J Clin Microbiol. 2015;53(5):1473-83.

38. Rufai SB, Kumar P, Singh A, Prajapati S, Balooni V, Singh S. Comparison of Xpert MTB/RIF with line probe assay for detection of rifampin-monoresistant Mycobacterium tuberculosis. J Clin Microbiol. 2014;52(6):1846-52.

39. Velayati AA, Farnia P, Mozafari M, et al. High prevelance of rifampin-monoresistant tuberculosis: a retrospective analysis among Iranian pulmonary tuberculosis patients. Am J Trop Med Hyg. 2014;90(1):99-105.

40. US Food and Drug Administration, 2013

41. Walker TM, Kohl TA, Omar SV, et al. Whole-genome sequencing for prediction of Mycobacterium tuberculosis drug susceptibility and resistance: a retrospective cohort study. Lancet Infect Dis. 2015;15(10): 1193-1202.

42. Chatterjee A, Nilgiriwala K, Saranath D, Rodrigues C, Mistry N. Whole genome sequencing of clinical strains of Mycobacterium tuberculosis from Mumbai, India: A potential tool for determining drug-resistance and strain lineage. Tuberculosis (Edinb). 2017;107:63-72.

43. Shea J, Halse TA, Lapierre P, et al. Comprehensive Whole-Genome Sequencing and Reporting of Drug Resistance Profiles on Clinical Cases of Mycobacterium tuberculosis in New York State. J Clin Microbiol. 2017;55(6):1871-1882.

44. Doughty EL, Sergeant MJ, Adetifa I, Antonio M, Pallen MJ. Culture-independent detection and characterisation of Mycobacterium tuberculosis and M. africanum in sputum samples using shotgun metagenomics on a benchtop sequencer. PeerJ. 2014;2:e585. 
45. Brown AC, Bryant JM, Einer-Jensen K, et al. Rapid Whole-Genome Sequencing of Mycobacterium tuberculosis Isolates Directly from Clinical Samples. J Clin Microbiol. 2015;53(7):2230-7.

46. Colman RE, Anderson J, Lemmer D, et al. Rapid Drug Susceptibility Testing of Drug-Resistant Mycobacterium tuberculosis Isolates Directly from Clinical Samples by Use of Amplicon Sequencing: a Proof-of-Concept Study. J Clin Microbiol. 2016;54(8):2058-67.

47. Votintseva AA, Bradley P, Pankhurst L, et al. Same-Day Diagnostic and Surveillance Data for Tuberculosis via Whole-Genome Sequencing of Direct Respiratory Samples. J Clin Microbiol. 2017;55(5):1285-1298.

48. Nelson KN, Shah NS, Mathema B, et al. Spatial Patterns of Extensively drug-resistant Tuberculosis (XDR-tuberculosis) transmission in KwaZulu-Natal, South Africa. J Infect Dis. 2018;

49. Guthrie JL, Delli pizzi A, Roth D, et al. Genotyping and Whole-Genome Sequencing to Identify Tuberculosis Transmission to Pediatric Patients in British Columbia, Canada, 2005-2014. J Infect Dis. 2018;218(7):1155-1163.

50. Sobkowiak B, Glynn JR, Houben RMGJ, et al. Identifying mixed Mycobacterium tuberculosis infections from whole genome sequence data. BMC Genomics. 2018;19(1):613.

51. Andries K, Verhasselt P, Guillemont J, et al. A diarylquinoline drug active on the ATP synthase of Mycobacterium tuberculosis. Science. 2005;307(5707):223-7.

52. Bryant JM, Harris SR, Parkhill J, et al. Whole-genome sequencing to establish relapse or re-infection with Mycobacterium tuberculosis: a retrospective observational study. Lancet Respir Med. 2013;1(10):786-92.

Received: January, 14, 2019

Accepted: March, 3, 2019 